\title{
Bimanual curvature discrimination of hand-sized surfaces placed at different positions
}

\author{
ABRAM F. J. SANDERS and ASTRID M. L. KAPPERS \\ Utrecht University, Utrecht, The Netherlands
}

\begin{abstract}
This study explores bimanual curvature discrimination of cylindrically curved, hand-sized surfaces. The setup was designed so that the postures of the observers' left and right arms and hands were the same as if the observers were holding a large object in their hands. We measured psychometric curves for observers who used active, dynamic touch; these curvatures ranged from 1.18 to $4.05 / \mathrm{m}$. Bimanual discrimination thresholds were found to be between 0.26 and $0.38 / \mathrm{m}$ on average; they were in the same range as unimanual thresholds reported in previous studies. Variation of (1) the horizontal distance between the stimuli or (2) the position of the setup had no effect on thresholds. In addition, we found that a number of observers showed discrimination biases in which they judged two physically different curvatures to be equal. Biases were of the same order of magnitude as the thresholds and could be either positive or negative. These biases can possibly be explained by small differences in left and right arm movements, an explanation that is supported by the position dependence of biases for individual observers.
\end{abstract}

Haptically acquired information about the shape of an object, the texture of its surface, its temperature, or its weight plays an important role in everyday manipulation of objects. Although we acknowledge that visual feedback most of the time makes a significant contribution, in many everyday actions such feedback is absent. Even with visual feedback present, afferent information can sometimes be essential, as is illustrated, for example, in the case study of a man suffering from a severe peripheral sensory neuropathy (Rothwell et al., 1982). Recently, a growing number of psychophysicists have turned to the topic of haptic perception of object properties. This article focuses on the perception of shape.

One valuable approach to the question of object properties that play a role in haptic shape perception is the use of natural and complex shapes. One could use familiar objects (as did, e.g., Klatzky, Lederman, \& Metzger, 1985), but perhaps more interesting are (haptically) unfamiliar objects, because of the absence of prior knowledge. What are the physical properties that an observer uses as cues to perceive an object's shape? Although there is a long history of vision research into this question, only few investigators have addressed it in haptics, mostly from the perspective of comparing the two modalities.

Norman, Norman, Clayton, Lianekhammy, and Zielke (2004) explored visual and haptic shape perception doing experiments in which observers had to compare the shapes of bell peppers. In one of the experiments, a bell pepper shape was manipulated haptically and matched to one of twelve visually presented shapes. Inspection of the objects

This research was supported by the Netherlands Organization for Scientific Research (NWO). Correspondence should be addressed to A. F. J. Sanders, Utrecht University, Helmholtz Instituut, P.O. Box 80000, 3508 TA Utrecht, The Netherlands (e-mail: a.f.j.sanders@phys.uu.nl). that had initially confused observers the most suggested that these objects had a similar global shape but differed in what one might call local features, such as "widths of trough gaps ... or large differences in surface curvature" (p. 347). The apparent increase of performance over time would then indicate that these local features gradually came into play and helped in matching the shapes. In any case, these results show that observers were able to mentally represent an object's overall shape, as well as the shape of characteristic substructures. Furthermore, their ability to quickly construct a representation of the overall shape without attending to local features suggests the use of global shape cues. However, the question of the nature of these cues remains.

The stimuli that Lakatos and Marks (1999) used (wooden objects modeling crystal structures) were categorized a priori by the experimenters on global and local geometrical shape features. Observers had to assess both visually and haptically the "overall degree of similarity between the shapes of [two] objects" (p. 897). In one of the experiments, the researchers investigated the effect of exploration time on similarity judgments both for pairs of objects that had similar global shapes but distinctive local features and for objects that differed in global shape and had no local features. Objects from the first set were judged to be more similar over time, whereas similarity judgments remained constant for the second set. Contrary to Norman et al.'s (2004) findings, these results suggest that local shape features are initially weighted more heavily, because they appear to have obscured the object's global shape.

This contradiction could probably be resolved by considering the characteristic differences between the stimulus sets. However, it should also be noted that the distinction between local and global shape features is somewhat ambiguous. For example, the additional local features in Lakatos and Marks's (1999) research were geometrically 
defined, but they could also interact perceptually with an object's global shape, as indeed appeared to be the case for some objects.

The most important thing these studies show, however, is that we still have little understanding of the physical object properties that observers may use as cues for perceiving shape in the first place. In both studies, only a sophisticated guess (based on visual inspection of the stimuli by the experimenters, and maybe observer interviews) could be made as to what the determining features were that an observer used to compare the shapes (although admittedly this was not the primary objective of the studies). Garbin and Bernstein (1984), for example, took a more systematic approach to this question. They had participants sort into groups a set of irregular shapes made from clay. The results of a multidimensional scaling analysis were then correlated with the ratings that participants gave on a number of physical object properties. Psychological measures were taken rather than physical measures, because of the difficulty of precise object quantification for complex forms.

Knowing the cues that play a role is one thing, but determining their psychophysical function is another. If observers indeed compare the curvatures of corresponding parts to match two shapes, what curvature differences are needed to allow the observers to perceive their dissimilarity? In other words, how large can the width of a trough gap be before the participant perceives its presence or before it starts changing the perceived overall shape? Because little haptic research has been devoted to psychophysical measurements of 3-D object properties (for a review, see Klatzky \& Lederman, 2003), we consider these important first steps to take when studying haptic shape perception, with the main challenge, of course, of physically quantifying the cues.

In our research, we therefore take a different approach to investigating the perception of object properties: By using stimuli that are mathematically well defined, we try to understand the important physical cues. The strength of our approach lies in our stimuli being real (physical) rather than virtual renderings (see, e.g., Henriques \& Soechting, 2003), which allows us to exploit the full range of cutaneous and kinesthetic abilities in a way similar to everyday exploration of natural objects. For reasons that will be explained below, curvature can be considered an important shape parameter. In this research, we mimicked everyday conditions as much as possible by looking at bimanual exploration, setting no restrictions on the way participants explored the stimuli, and designing the setup so that the postures of the observers' arms and hands were the same as if they were holding an object in their hands.

Many objects we encounter in daily life are bounded by smooth surfaces. Even when an object is not completely smooth, its surface usually consists of smooth parts that are interrupted by ridges and edges. For any smooth object, we can approximate the local surface around any of its points in terms of doubly curved surfaces (Koenderink \& van Doorn, 1992). Such a doubly curved surface is fully defined by the "curvatures" along two orthogonal axes. Since these curvatures are independent of the position of the object in space, they make good candidates for an in- trinsic description of shape. We can then characterize the shape of any three-dimensional object in a sufficiently small neighborhood by specifying the two orthogonal curvatures. For that reason, curvature is an important parameter in the study of haptic shape perception.

Consider the example of a sphere (e.g., a basketball). The two orthogonal curvatures of the local surface patch are both equal to the reciprocal of the radius of the sphere. This is intuitively clear, for it is easily understood that the surface of a sphere has a constant curvature that decreases for spheres with larger radii. In this article, we deal with curved surfaces of circular cylinders. Like the sphere, any point on the surface of such a cylinder has the same local curvature, which makes it a suitable object for experiments on curvature perception. The curvature of the surface along the cylinder axis is zero, whereas the curvature in the perpendicular direction is equal to the reciprocal of the radius. In a sense, one might call this a one-dimensional curvature. Note that curvature is not only a measure of shape, but also of size: Most people would say that a wine bottle and a beer bottle have the same shape, although the surface of the beer bottle has greater curvature.

A number of psychophysical studies have investigated unimanual curvature discrimination: Observers had to discriminate between two curved profiles by touching them with one finger or hand successively. Discrimination thresholds were measured for different curvature profiles, stimulus dimensions, and placements on the hand, and for static and dynamic touch. So far, however, little attention has been paid to bimanual curvature discrimination, in which observers discriminate two curvatures by touching them with two hands simultaneously. For example, Kappers and Koenderink (1996) reported that in experiments with cylindrically curved surfaces, performance was better for unimanual than for bimanual discrimination. However, they did not measure psychometric curves and gave no values for discrimination thresholds. In our research, we will focus on bimanual curvature discrimination, but first we will give a short overview of the research on unimanual discrimination that is relevant to our study.

One of the first studies to report systematic measurements of unimanual discrimination thresholds was by Gordon and Morison (1982). Observers explored small strips of lengths varying from 2 to $4 \mathrm{~cm}$ with the tip of their index finger. For example, the authors found that observers could discriminate between two 2-cm strips with base-to-peak heights of only 0.12 and $0.22 \mathrm{~mm}$. More extensive research was done by Pont, Kappers, and Koenderink (1997). They investigated static discrimination of cylindrically curved, 20 -cm-long strips placed in nine different positions on the palmar and dorsal sides of the hand. Discrimination thresholds were found to be significantly higher in the dorsal condition. For all positions on the palmar side of the hand, thresholds ranged between 0.22 and $1.48 / \mathrm{m}$ (reference curvatures were $-0.8,0$, and $+0.8 / \mathrm{m}$ ). Pont et al. (1997) argued that because cutaneous resolution is much lower on the dorsal side, the result indicated that cutaneous stimulation makes an important contribution to the discrimination of curved strips. 
In a follow-up study, Pont, Kappers, and Koenderink (1999) compared static and dynamic touch and determined the effective stimulus for unimanual curvature discrimination. Discrimination was tested under different conditions for cylindrically curved strips of lengths varying from 5 to $20 \mathrm{~cm}$. A surprising result was the quantitative similarity of thresholds for static and dynamic touch, despite the additional component of self-controlled exploratory movements in dynamic discrimination. They also found similar mechanisms underlying both static and dynamic curvature discrimination. Discrimination performance in the experiments by Pont et al. (1999) could be explained by an attitude comparison model with an incomplete correction for stimulus length. For example, in the case of static touch with one finger, participants discriminated successively presented stimuli by comparing the local slopes. When observers used two or three fingers, attitude differences over the surfaces between the two outermost fingers were compared. Larger attitude differences then corresponded to more curved strips. In the case of dynamic touch, the effective stimuli were again attitude differences. If curvature discrimination were based only on attitude comparison and the distance between the fingers were ignored, observers would underestimate the curvature of shorter strips in comparison with longer strips with the same curvature. Indeed, Pont et al. (1999) found that observers made systematic errors, but these errors were not as large as expected. Apparently, curvature judgments were not completely independent of stimulus length.

Another relevant study concerning unimanual discrimination was conducted by Louw, Kappers, and Koenderink (2000). They determined discrimination thresholds (discrimination from flat) for Gaussian-shaped profiles covering a wide range of spatial scales. The length of the strips varied from 4 to $90 \mathrm{~cm}$. Over the entire range, Louw et al. found the same dependence of threshold amplitudes on the spatial width of the Gaussian profile; this result is surprising, considering that in this range of scales different cutaneous and kinesthetic mechanisms contribute to haptic perception. Louw et al. explained that the result is in close agreement with conclusions reached by Pont et al. (1999) concerning the effective stimulus for curvature discrimination.

\section{The Present Study}

The present research focuses on bimanual curvature discrimination. There are a number of questions we hope to answer in this study. First, we want to know how unimanual and bimanual discriminatory sensitivities relate. Threshold measurements enable us to compare these two conditions. One could hypothesize that given the sequential nature of unimanual exploration, bimanual discrimination should outperform the unimanual condition. On the other hand, bimanual discrimination requires the integration of bilateral nerve signals in the central nervous system, which might pose a disadvantage for the bimanual condition.

It is natural for observers to use two hands instead of one when handling objects that are relatively large. In this study, we therefore used hand-sized stimuli. We oriented the stimuli in such a way that the postures of the observers were the same as if they were holding an object in their hands (see Figure 1). The observers were free to explore the stimuli in whatever way they preferred, and the stimuli were large enough for them to use the entire hand to touch the surfaces. The next question, therefore, concerned the effect of surface area. Depending on the exploration strategy applied, an increase in surface area might give an observer increased skin contact area (thus, additional cutaneous information) and induce additional arm movements up to the shoulder (additional kinesthetic information), relative to the exploration of curved strips. In the case of hand-sized surfaces, thresholds either decrease or remain constant when redundant information is available.

Although the shape of an object is independent of its position, it might be more difficult to perceive shape when an object is not right in front of the observer but is held to the left, to the right, or even above the head. It might also be more difficult to perceive the shape of two objects that are farther apart. In this study, we asked ourselves whether the positioning of the stimuli influences discrimination performance. We built a setup with which we could vary the positions of two stimuli in front of the observer independently on a horizontal line from left to right. We varied the distance between the stimuli, keeping them positioned symmetrically with respect to the observer (Experiment 2), and we varied the position of the stimulus set, placing it to the left, in front, and to the right of the observer (Experiment 3).

The study by Kappers and Koenderink (1996) mentioned above also reported that the placement order of the stimuli had an effect on discrimination in the bimanual condition. For 3 out of 4 observers, discrimination performance was much better when the more curved surface was placed on either the left or the right. Which placement order resulted in best performance depended on the observer. A response bias was ruled out as a possible explanation for the observed asymmetry, because both the bimanual and unimanual conditions would then have been influenced to the same extent, which was not the case. Kappers, Koenderink, and te Pas (1994) reported a similar finding.

The placement order effect reported in these two studies provides strong evidence that bimanual curvature discrimination may be biased. Suppose, for example, that an observer overestimates the curvature that is presented to the right hand. If in a bimanual discrimination experiment the right stimulus is (physically) more curved, discrimination improves, because the observer perceives an even larger curvature difference between the two stimuli. Correct response scores go up, resulting in a shift of the psychometric curve. In the reverse order, however, discrimination performance is worse. The final motivation for the present study is, therefore, to investigate whether bimanual discrimination can indeed be biased. Does the left or the right hand systematically overestimate curvature? The studies by Kappers and Koenderink (1996) and Kappers et al. (1994) suggest that different observers can 
have different behavior, since some showed and others did not show a placement order effect. What is more, for different observers in those experiments the effects were in opposite directions. However, it should be noted that these studies were carried out with only a few observers.

\section{GENERAL METHOD \\ Experiments 1, 2, and 3}

\section{Stimuli}

The stimuli in this study had a length and width of $29 \mathrm{~cm}$ and a constant peak height of $5 \mathrm{~cm}$. They were made from polyurethane foam filled with an artificial resin. The top surface was cylindrically curved. The reciprocal of the radius of the corresponding cylinder was taken to be the curvature of the stimulus. We produced stimuli with a radius of curvature between 24.7 and $84.7 \mathrm{~cm}$; this covered the range of curvatures that was physically possible with these stimulus dimensions. Consecutive stimuli differed by a constant increase in radius of $50 \mathrm{~mm}$. The stimuli had the following curvatures: $1.18,1.25,1.34$, $1.43,1.55,1.68,1.83,2.01,2.24,2.52,2.88,3.37$, and $4.05 / \mathrm{m}$. Two stimuli were available for each curvature. In Figure 1A, the most and least curved test stimuli are shown as viewed from the side.

\section{Experimental Setup}

The observers were seated behind a small table and blindfolded to prevent them from seeing the stimuli. We put a heavy metal support with a rail and two metal stimulus holders on top of the table, the center of the rail about $30 \mathrm{~cm}$ from the table's edge. The stimulus holders could be moved independently on the rail in front of the observer from left to right, and vice versa. Stimuli were easily fixed onto and removed from the two stimulus holders. The curved side of the left stimulus pointed outward to the left, the curved side of the right stimulus to the right; the cylinder axis of the stimulus was oriented horizontally. A small hole in the middle of the table enabled the observers to check their positions during the experiments. Figure 1 shows three pictures of the setup, with the stimuli in different positions in each of them. Note that in the experiments, curvature differences were much smaller than those shown in panel A; we used stimulus pairs like the one shown in panels B and C.

\section{Procedure}

The experiments were designed according to the two-alternative forced choice method. In each trial, two stimuli were presented at the same time: The left stimulus was touched by the left hand, the right one by the right hand. The observers were asked to indicate which stimulus they judged to be more curved and were allowed to answer only "left" or "right." A trial started after the experimenter had put both stimuli into position. The participants were free to explore the surfaces in whatever way they preferred, but they were not allowed to touch other sides of the stimuli than the curved surfaces. In effect, all observers used their entire hands, including the palms, to scan the stimuli by moving their hands systematically up and down over the surfaces. The only exception was observer M.H., who used only her fingers to explore the stimuli. No observer used static hand positioning exclusively. Some observers participated in more than one experiment, which they performed in a random order.

\section{Analysis}

For each combination of stimuli, we computed (1) the curvature difference, defined as the curvature on the left (which was either the reference or the test stimulus) minus the curvature on the right (either the test or the reference stimulus), and (2) the fraction of all ten repetitions in which the observer judged the left stimulus to be more curved. Cumulative Gaussian distributions were used as psychometric functions and were fitted to the data on a linear scale by means of a maximum-likelihood estimate (see the Appendix). A curve consisted of 14 data points, each one sampled ten times.
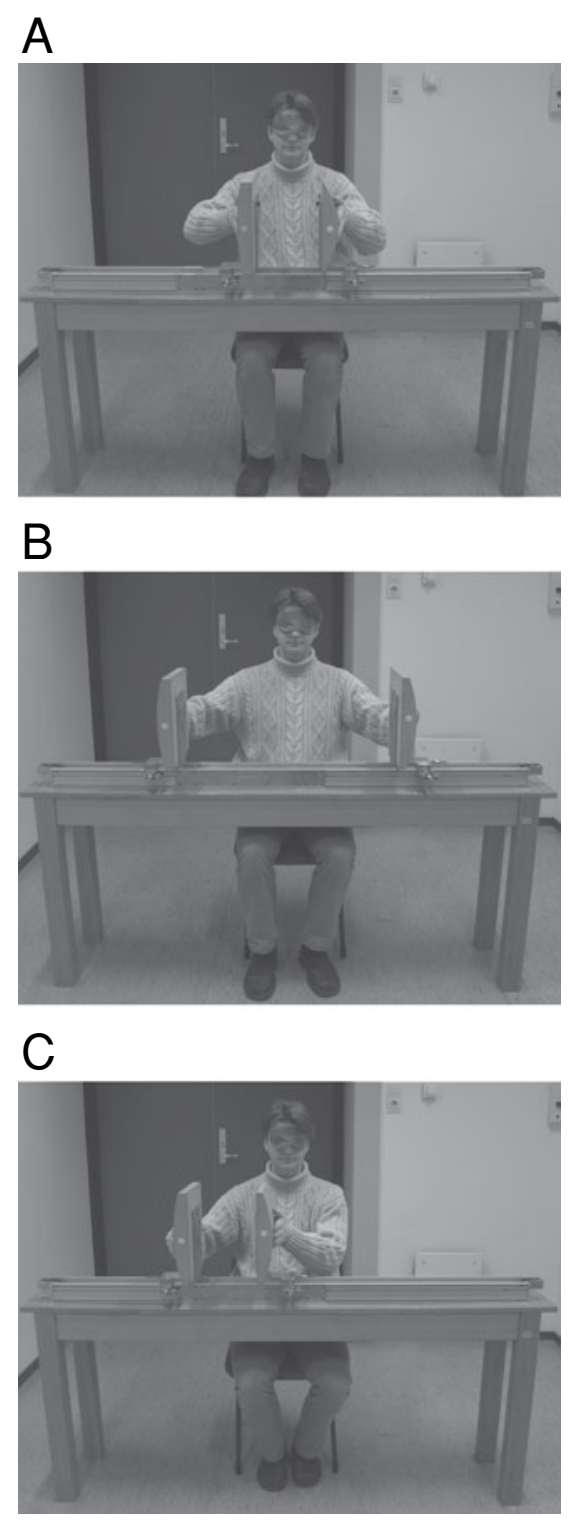

Figure 1. An observer trying to feel which of the two surfaces is more curved. Panel A shows the setup in Experiment 1; panels $B$ and $C$ show two examples of setups in Experiments 2 and 3, respectively. Note that in panel $A$, the most curved and the flattest stimulus are shown, but in the experiments curvature differences were much smaller.

The fitted psychometric curves are characterized by two parameters, which will be denoted by $\mu$ and $\sigma$ (see Figure 2). Parameter $\mu$ (mean) represents the curvature difference at which the observer decided in $50 \%$ of all trials that the left stimulus felt more curved. A nonzero mean indicated that the observer showed a bias; two stimuli that had physically different curvatures gave rise to a response score of $50 \%$. Parameter $\sigma$ is inversely related to the steepness of the curve. We define the discrimination threshold to be the curvature difference that yielded a rise from $50 \%$ to $84 \%$ in the response score. In that case, the discrimination threshold is represented by $\sigma$. Figure 2 depicts a representative example of psychometric curves obtained in this study. We estimated standard errors in fitted parameters $\mu$ and $\sigma$ using a parametric bootstrap procedure (see the Appendix). 


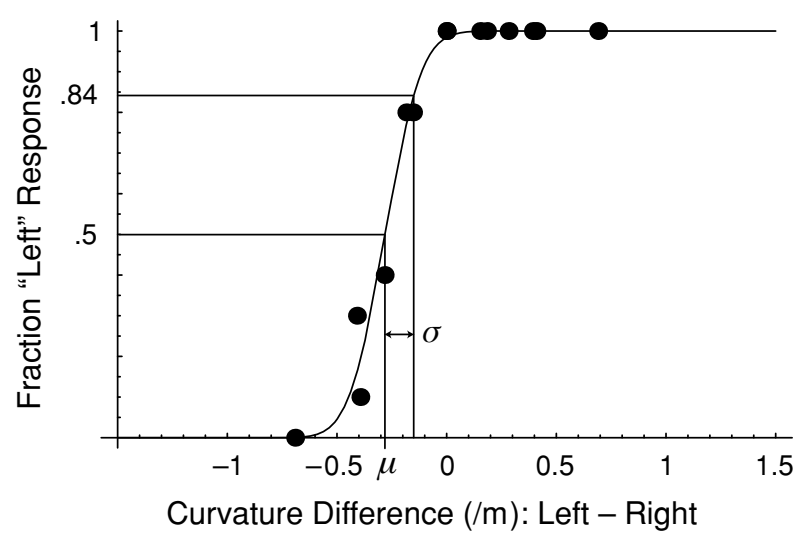

Figure 2. The fraction of trials in which the observer responded that the left stimulus was more curved is plotted as a function of the curvature difference between the two stimuli. A cumulative Gaussian function is fitted to the data.

\section{EXPERIMENT 1}

In the first experiment, we measured bimanual discrimination thresholds and biases for three different reference curvatures to find out more about the curvature dependence of these parameters.

\section{Method}

Participants. Ten undergraduate students from the Department of Physics and Astronomy at Utrecht University participated in Experiment 1 . According to Coren's (1993) questionnaire, they were all right handed; 3 of them were female (C.D., D.L., and M.H.). All observers were naive with regard to the aims and design of the experiment and were paid for their efforts. Some observers also participated in Experiment 2 and/or Experiment 3 at an earlier or a later time.
Procedure. We used three reference stimuli with curvatures 1.43, 1.83 , and $2.52 / \mathrm{m}$ and combined each of them with seven test curvatures: Three curvatures were larger than, three smaller than, and one was the same as the standard stimulus. For example, a reference curvature of $1.43 / \mathrm{m}$ was combined with curvatures $1.18-1.83 / \mathrm{m}$. The stimuli were positioned symmetrically with respect to the observer (see Figure 1A); the distance between the two peaks of the stimuli was $30 \mathrm{~cm}$. Reference stimuli were presented to both the left and right hands; each combination was repeated ten times. For a single observer, this amounted to 420 trials ( 3 reference curvatures $\times 7$ test curvatures $\times 2$ reference positions $\times 10$ repetitions). Trials were randomized in the following way: All 420 trials were divided into ten blocks of 42 trials in which each combination was presented once; all blocks were randomized independently. The experiment was divided into sessions of $45 \mathrm{~min}$; the entire experiment took about 5-6 h for each observer. Sessions were generally separated by at least 1 day. The observers received no feedback.

\section{Results}

Figure 3 depicts bimanual discrimination thresholds $(\sigma)$ as a function of reference curvature for all observers in the first experiment. Average discrimination thresholds were $0.26,0.32$, and $0.38 / \mathrm{m}$ for reference curvatures 1.43 , 1.83 , and $2.52 / \mathrm{m}$, respectively; thresholds ranged between 0.16 and $0.54 / \mathrm{m}$. Thresholds and corresponding standard errors correlated strongly: The standard error was $16 \%$ of $\sigma$, on average. To avoid cluttering the graph, we did not indicate errors in Figure 3.

A one-way ANOVA with a repeated measures design showed a significant main effect of reference curvature $[F(2,18)=15.28, p<.001]$. To further analyze the effect of reference curvature, we performed paired $t$ tests. Compared to the Bonferroni-corrected significance level $(\alpha)$ of .017, we found that all thresholds differed significantly from each other $[0.26-0.32 / \mathrm{m}, t(9)=2.95, p=.016$; $0.26-0.38 / \mathrm{m}, t(9)=4.80, p=.001 ; 0.32-0.38 / \mathrm{m}, t(9)=$ $3.11, p=.013]$.

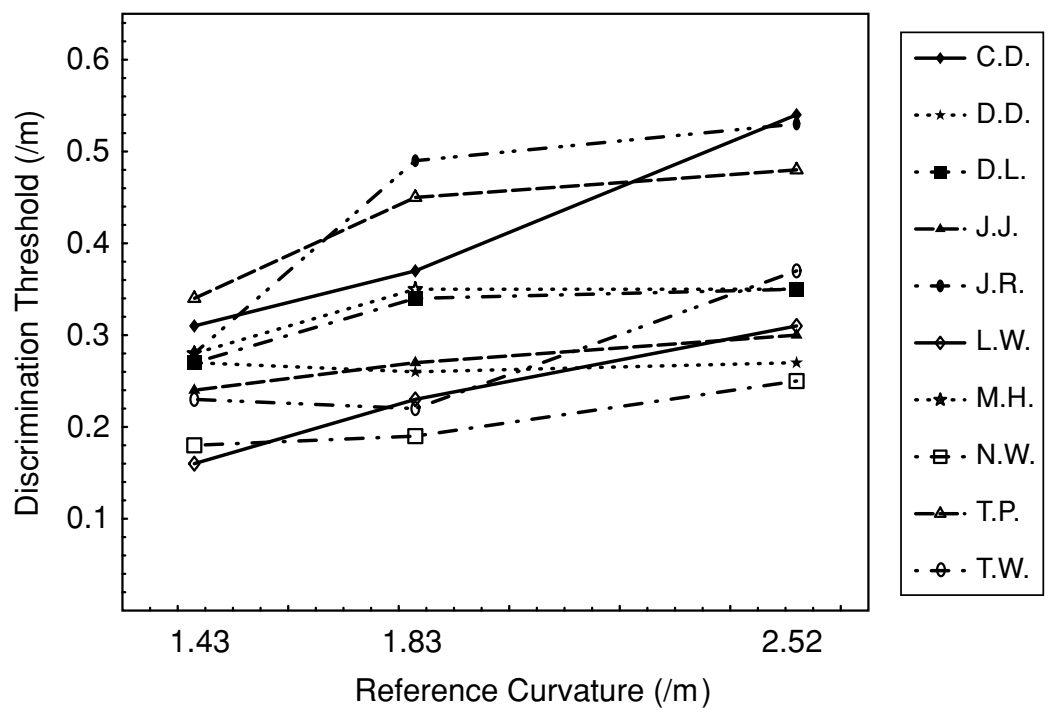

Figure 3. Discrimination thresholds against reference curvature for all 10 observers participating in Experiment 1. 

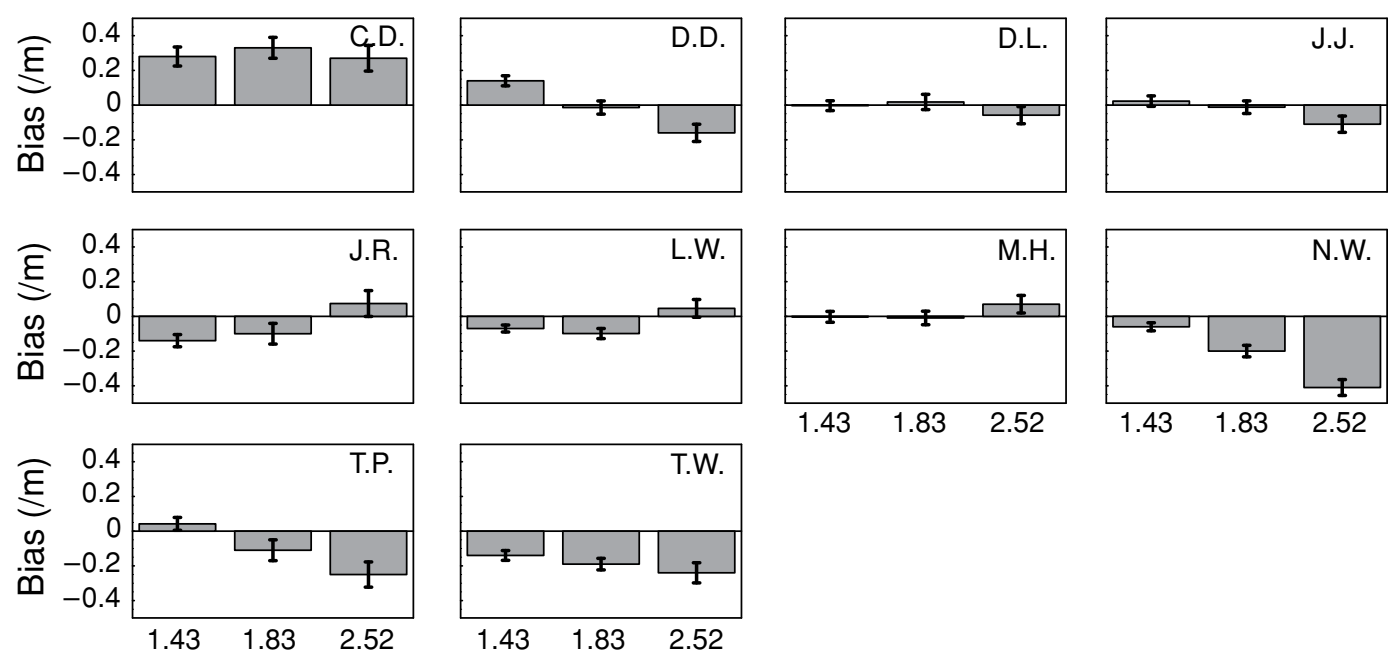

Reference Curvature (/m)

Figure 4. Biases against reference curvature (Experiment 1); standard errors are indicated.

The increase of thresholds with reference curvature suggests that curvature discrimination is subject to Weberlike behavior. Therefore, we computed Weber fractions by dividing thresholds by reference curvature to see if they were constant. The average fractions were $18 \%, 17 \%$, and $15 \%$ for the reference curvatures $1.43-2.52 / \mathrm{m}$. Although a one-way ANOVA with a repeated measures design did reveal a significant effect of curvature $[F(2,18)=5.05, p<$ $.025]$, paired $t$ tests, with a Bonferroni correction lowering the significance level $\alpha$ to .017 , indicated that only the fractions $18 \%$ and $15 \%$ differed significantly [ $18 \%-17 \%$, $t(9)=-0.55, p>.5 ; 18 \%-15 \%, t(9)=-3.00, p=.015$; $17 \%-15 \%, t(9)=-2.55, p=.03]$.
Figure 4 gives separate graphs for each observer, representing biases $(\mu)$ and corresponding standard errors. Close inspection of Figure 4 reveals that for some observers (e.g., N.W.) standard errors were small relative to biases: Their biases were significantly nonzero. For other observers, a zero bias fell within one standard error (e.g., D.L.). Some participants showed positive biases (e.g., C.D.), and others showed negative biases (e.g., T.W.). We did not analyze the results in more detail because it was apparent from Figure 4 that biases were strongly participant dependent. Finally, it should be noted that for a few individual observers, biases seemed to depend on curvature.

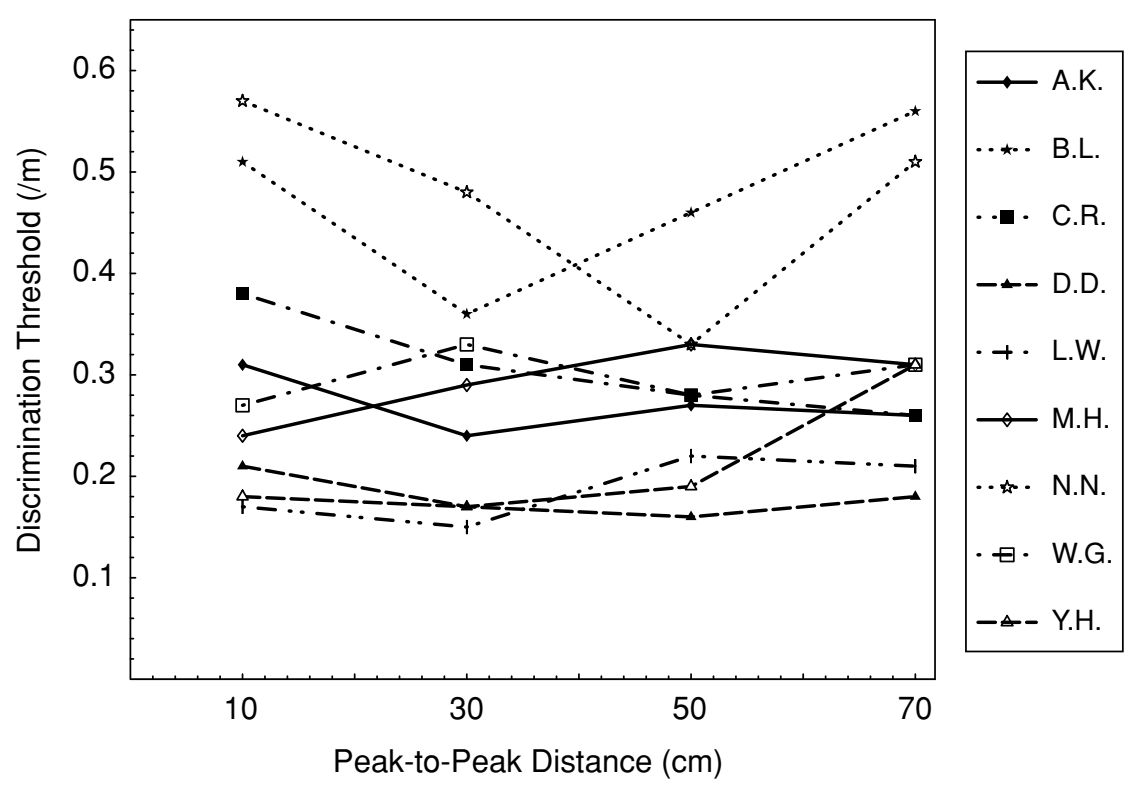

Figure 5. Discrimination thresholds against peak-to-peak distance for all 9 observers in Experiment 2. 


\section{EXPERIMENT 2}

In Experiment 2, we varied the distance between the two stimuli, keeping them positioned symmetrically with respect to the observer. In one trial, the postures of the left and right hands and arms were the same.

\section{Method}

Participants. Ten undergraduate students from the Department of Physics and Astronomy at Utrecht University participated in this second experiment. Of these 10, 4 were men (B.L., D.D., L.W., and W.G.); only C.R. was left handed according to the Coren (1993) questionnaire. The observers were all naive and were paid for their efforts. Some observers also participated in Experiment 1 and/or Experiment 3 at an earlier or a later time.

Procedure. The procedure was the same as in the first experiment. This time, however, we used only one reference stimulus, with curvature $1.83 / \mathrm{m}$, combined it with seven test stimuli $(1.43-2.52 / \mathrm{m})$, and varied the peak-to-peak distance. The peak-to-peak distance is defined as the distance between the peaks of the two stimuli. The stimuli were always positioned symmetrically with respect to the observer. We measured psychometric curves for four peak-to-peak distances: 10, 30, 50, and $70 \mathrm{~cm}$ (Figure 1B shows the setup for a peak-to-peak distance of $70 \mathrm{~cm}$ ). This amounted to 560 trials for each observer: 1 reference curvature $\times 7$ test curvatures $\times 2$ reference positions $\times 4$ distances $\times 10$ repetitions. The trials were randomized in the following way: All ten repetitions were randomized independently and measured successively. One repetition consisted of four sublists of 14 trials, one for each separation distance. Within each sublist, all 14 trials were randomized; the order in which the four sublists were presented was also randomized. The entire experiment took about $6-7 \mathrm{~h}$ for each observer to complete.

\section{Results}

The psychometric curves for 1 particular observer did not fit the data well, so we excluded her results from our analysis.

Figure 5 shows thresholds as a function of the peakto-peak distance for 9 observers. The overall average dis- crimination threshold was $0.30 / \mathrm{m}$; thresholds ranged from 0.15 to $0.57 / \mathrm{m}$. Standard errors were approximately $16 \%$ of $\sigma$. As expected from Figure 5, a one-way ANOVA with a repeated measures design revealed no significant main effect of peak-to-peak distance on thresholds $[F(3,24)=$ $1.65, p>.10]$.

In Figure 6, we have plotted discrimination biases against peak-to-peak distance for every observer separately. For most observers, errors were small compared with biases (e.g., D.D., Y.H.). Observer B.L. showed mainly zero biases. Other observers had a nonzero bias for a particular peak-to-peak distance, whereas the bias disappeared for other peak-to-peak distances. It is clear from Figure 6 that there was no general dependence of biases on peak-to-peak distances. Note that for some of the observers, the graphs suggest that biases increased or decreased with peak-to-peak distance.

\section{EXPERIMENT 3}

In Experiment 2, stimuli were always positioned symmetrically with respect to the observer. In the third experiment, we wanted to investigate whether an asymmetrical positioning of the stimuli, causing different postures of the left and right arms in one trial, would influence performance.

\section{Method}

Participants. Ten students from the Department of Physics and Astronomy at Utrecht University participated in this experiment. None of them was left handed according to the Coren (1993) questionnaire, and 4 were women (B.P., M.H., N.N., and Y.H.). All participants were naive as to the designs and aim of the experiment. Some observers also participated in Experiment 1 and/or Experiment 2 at an earlier or a later time.

Procedure. The procedure was similar to that in Experiment 2. We combined a reference stimulus with curvature $1.83 / \mathrm{m}$ with seven
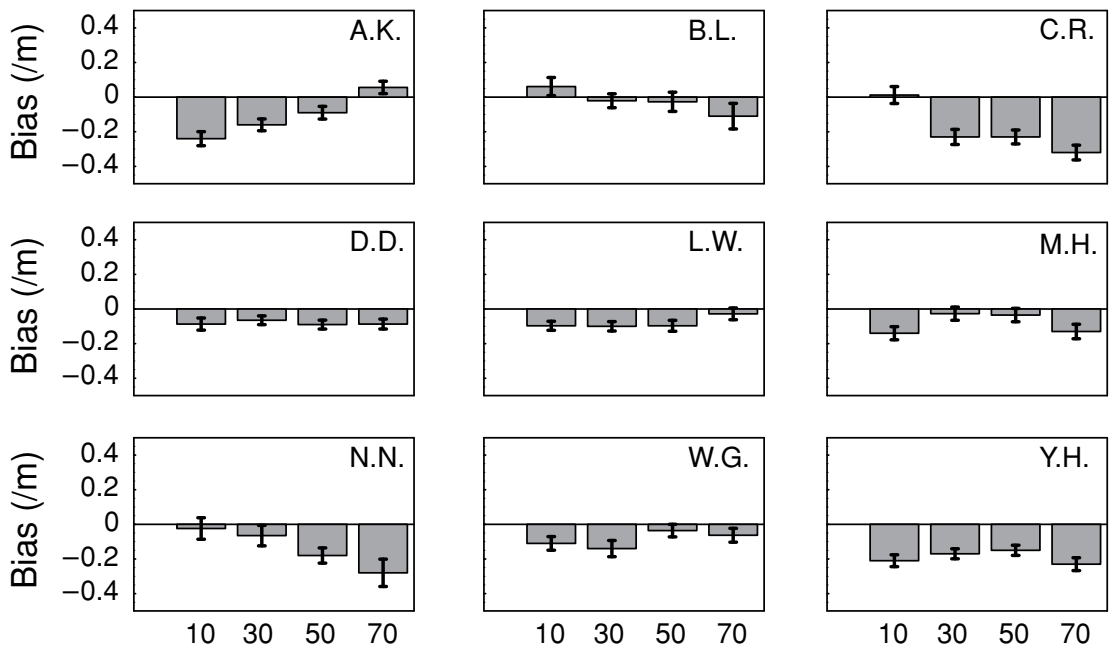

Peak-to-Peak Distance (cm)

Figure 6. Biases against peak-to-peak distance (Experiment 2); standard errors are indicated. 
test stimuli (curvatures $1.43-2.52 / \mathrm{m}$ ) but kept the peak-to-peak distance between the stimuli at $30 \mathrm{~cm}$. The center point of the stimulus set (the point between the two stimuli) was positioned 20 or $10 \mathrm{~cm}$ to the left of the observer, in front of him or her, or 10 or $20 \mathrm{~cm}$ to the right. Positive center positions correspond to a positioning of the stimuli to the right of the observer; negative positions to the left. Figure $1 \mathrm{C}$ shows the setup for a center position of $20 \mathrm{~cm}$. Each observer did 700 trials ( 1 reference stimulus $\times 7$ tests $\times 2$ hands $\times$ 5 center positions $\times 10$ repetitions). Trials were randomized in the following way: All ten repetitions were randomized independently. A repetition consisted of five sublists of 14 trials, one for each center position. Within each sublist, all 14 trials were randomized; the order in which the five sublists were presented was also randomized. Each observer took about 7-9 $\mathrm{h}$ to complete the entire experiment.

Analysis. Probably because the psychometric curves for observer N.N. were very steep, we did not succeed in fitting the curves using a maximum-likelihood procedure. For this observer, we used a leastsquares method.

\section{Results}

Figure 7 depicts discrimination thresholds as a function of center position for 10 observers. The overall average discrimination threshold was $0.22 / \mathrm{m}$; thresholds ranged from 0.02 to $0.40 / \mathrm{m}$. We do not indicate standard errors on the figure, but they were approximately $16 \%$. As expected from Figure 7, a one-way ANOVA with a repeated measures design showed no significant effect of center position $[F(2.19,19.73)=1.14, p>.25]$.

Figure 8 shows biases and standard errors for each observer. The results for most observers are very clear: Some had nonzero biases (e.g., J.H., T.W.), and others had zero biases (e.g., A.H., N.N.). Obviously, there was no general dependence of biases on center positions. For several observers, biases seem to have increased or decreased with center position (e.g., N.W., W.B.).

\section{EXPERIMENT 4}

If an observer's response score is $50 \%$, we assume that the observer perceives no curvature difference between the two stimuli. For that reason, the mean of the psychometric curve is sometimes appropriately called the point of subjective equality. However, it is possible for an observer to show a response bias, which means that response is biased toward one of the response alternatives, irrespective of the stimulus intensity (Macmillan \& Creelman, 1991). Consequently, the subjectively equal curvature pair then corresponds to a response score that is either above or below $50 \%$, depending on whether the response is biased toward "left" or "right," respectively. In Experiment 4, we did matching experiments with 5 observers who had participated in one or two of the previous experiments. It was the observers' task to match the reference curvature with a test stimulus that they perceived as having the same curvature. This procedure enabled us to measure the subjectively equal stimulus pair directly.

\section{Method}

Participants. Five observers participated in this experiment, all of whom had been observers in one or two of the previous three experiments: B.L. (Experiment 2), J.H. (Experiment 3), A.H. (Experiment 3), T.W. (Experiments 1 and 3), and Y.H. (Experiments 2 and 3).

Stimuli and Experimental Setup. The stimuli and the setup were the same as in the previous three experiments.

Procedure. All three discrimination experiments had one common condition - that is, a reference curvature of $1.83 / \mathrm{m}$, a peak-topeak distance of $30 \mathrm{~cm}$, and a center position of $0 \mathrm{~cm}$. In Experiment 4 , we determined the subjectively equal stimulus pair for the same experimental condition. At the beginning of a new matching

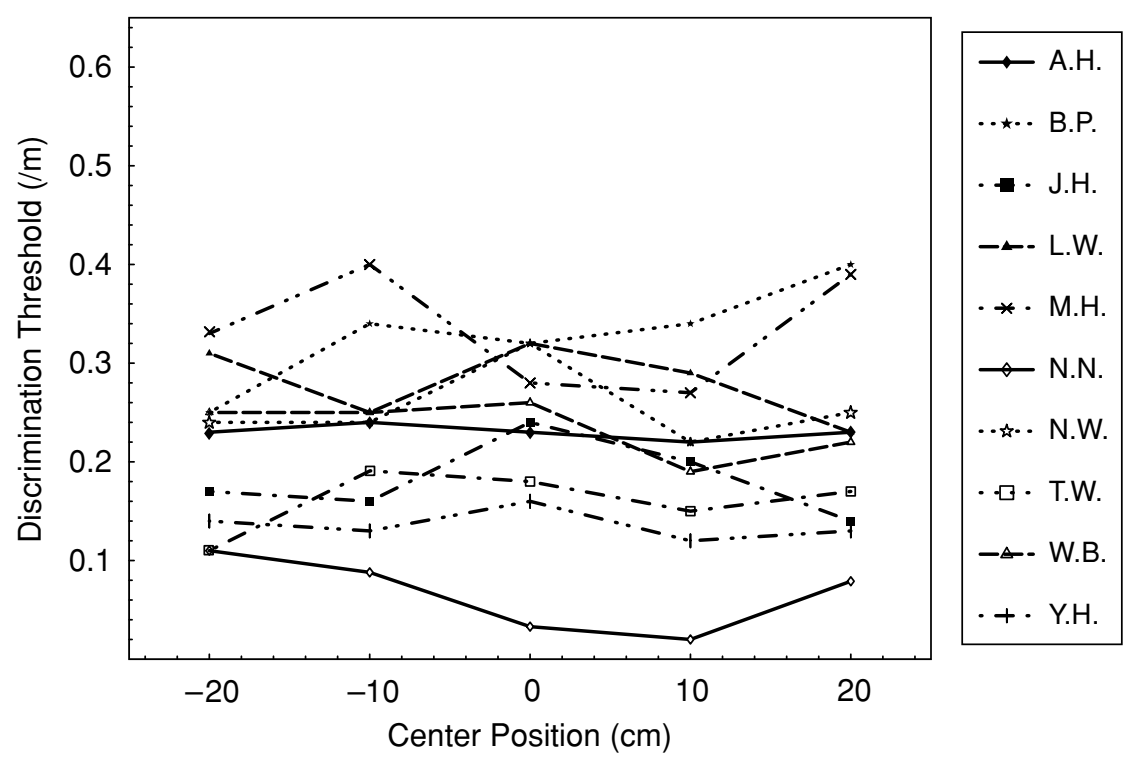

Figure 7. Discrimination thresholds against center position for all 10 observers in Experiment 3. 

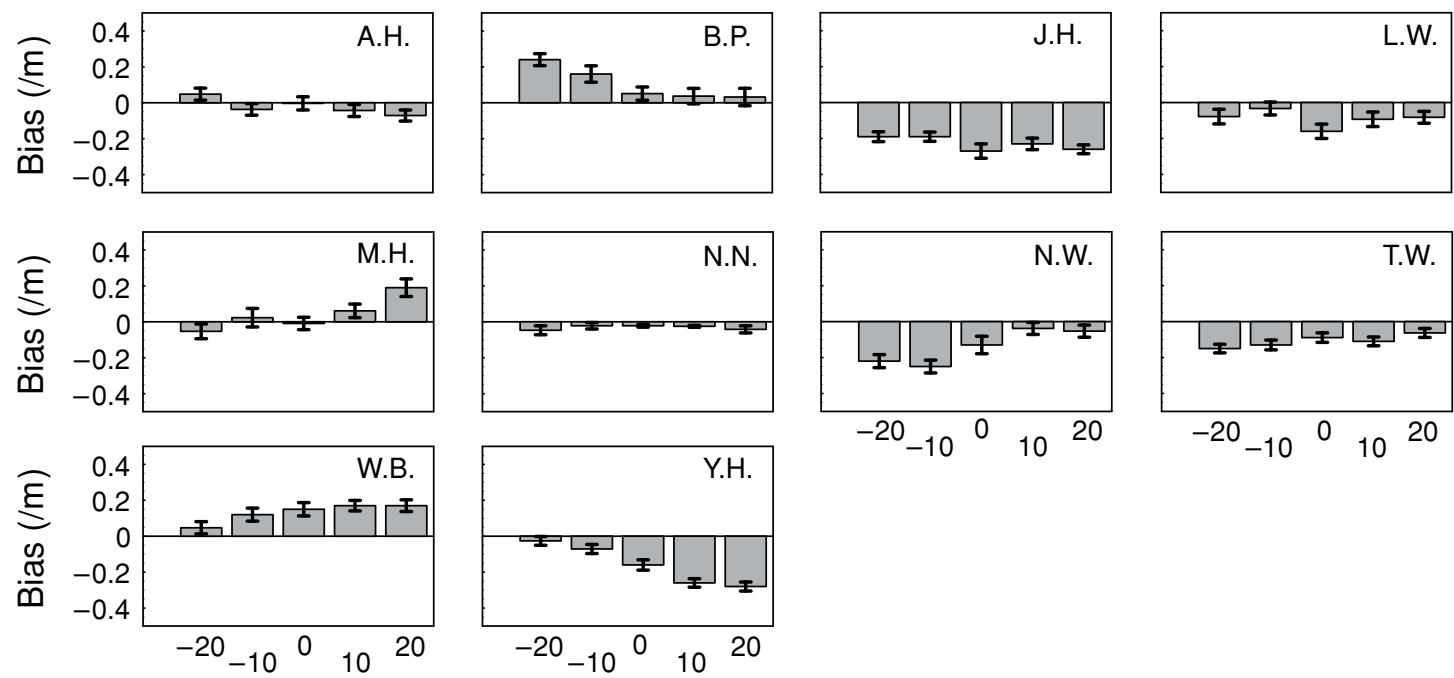

Center Position (cm)

Figure 8. Biases against center position (Experiment 3); standard errors are indicated.

series, we informed the observer on which side we had positioned the reference stimulus. A trial started after the experimenter had put both the reference and test curvatures in position. After each trial, the observer asked for a more curved or less curved test stimulus. This process continued until the observer decided that both the reference and test curvatures felt the same. There were no time constraints. Each of the two reference positions was sampled six times. We picked the first test curvature randomly from the stimuli with curvatures $1.25-3.37 / \mathrm{m}$; the order of all 12 trials was also randomized. It took each observer $45 \mathrm{~min}$ to $1 \mathrm{~h}$ to complete the experiment.

Analysis. For every observer, we calculated both the mean and the standard deviation of the curvature differences (left minus right) between the matched stimuli.

\section{Results}

For each observer, Figure 9 depicts the bias found in the matching experiment against the bias measured in the discrimination experiment for the same experimental condition. For the 2 observers who had participated in two discrimination experiments, we computed the weighted average of the two biases. Standard errors are also indicated in Figure 9. The dashed line is the $y=x$ line; points on this line represent observers who had exactly the same bias in both the matching and discrimination experiments. Observers J.H., T.W., and Y.H. all had nonzero, negative biases in

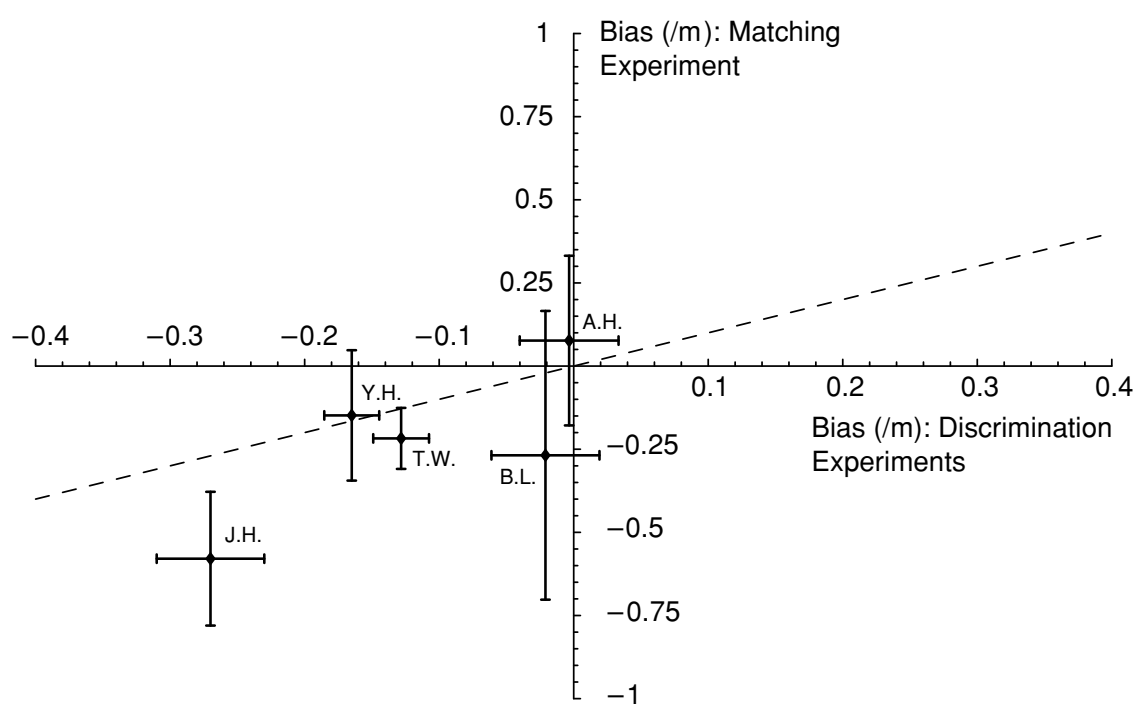

Figure 9. Biases measured in the matching experiment (Experiment 4) against biases measured in the discrimination experiments for 5 observers. Standard errors are indicated. 
the discrimination experiments. The first 2 also showed nonzero biases in the fourth experiment, but for observer Y.H. a zero bias fell just within the standard error. Observers A.H. and B.L. showed zero biases in the matching experiment as well as in the discrimination experiment.

\section{DISCUSSION AND CONCLUSIONS}

We measured curvature discrimination thresholds and biases for hand-sized stimuli and varied curvature and spatial location in four experiments. Observers combined both tactile and kinesthetic sensations from active exploratory movements in a way similar to everyday manipulation of objects. We now discuss the results in more detail.

\section{Discrimination Thresholds}

Close inspection of Figures 3, 5, and 7 shows that thresholds were in the same range for all observers. We found a significant increase of thresholds with reference curvature in the curvature range from 1.43 to $2.52 / \mathrm{m}$, suggesting that bimanual discrimination is subject to Weberlike behavior. Consequently, we would expect Weber fractions $(18 \%, 17 \%$, and $15 \%$ on average) to be constant, but in a statistical analysis we could not decide whether this was indeed the case. Goodwin and Wheat (1992) reported thresholds for much larger curvatures and, consequently, much smaller stimuli. They studied passive discrimination of spherically curved surfaces applied to the finger pad. Their thresholds (at 75\% correct) were $18 \%$ for a reference curvature of $154 / \mathrm{m}$ and $13 \%$ for $286 / \mathrm{m}$. Although the studies were conducted under different conditions, Goodwin and Wheat's results together with ours indicate that Weber fractions might indeed be similar across a wide range of curvatures.

\section{Bimanual Versus Unimanual Discrimination}

One of the motivations for this study was to compare the results for bimanual discrimination with unimanual curvature discrimination thresholds reported in previous studies. Although limited data on unimanual discrimination are available, we know of two studies for which the experimental conditions resemble those of our experiment. Both studies measured thresholds for hand-sized stimuli that were being touched with the entire hand. Vogels, Kappers, and Koenderink (1996) reported curvature detection thresholds for spherically curved stimuli, and these thresholds averaged $0.23 / \mathrm{m}$ over 3 participants. Vogels, Kappers, and Koenderink (1999) used doubly curved surfaces and asked observers to discriminate from a flat plane the curvature along a specified orientation. Discrimination thresholds in that study were between 0.20 and $0.32 / \mathrm{m}$ on average for 7 observers. In these studies, observers used static touch only, whereas in our experiment stimuli were explored dynamically. However, Pont et al. (1999) found that, at least for discrimination of curved strips, static and dynamic discrimination thresholds were similar.

Thus we see that, in comparison with the thresholds obtained in our study $(0.26-0.38 / \mathrm{m})$, unimanual thresholds were in the same range as thresholds in the bimanual con- dition. We found no indications that unimanual discrimination performance differs from bimanual discrimination performance. The supposed disadvantage of integrating bilateral signals in bimanual discrimination does not outweigh the drawback of the additional memory component in the unimanual case. Apparently, the ability to discriminate is the same whether two curvatures are touched with two hands simultaneously or with one hand successively.

\section{Contact Area}

Our observers were free to explore the surfaces in whatever way they preferred. However, all of the observers used dynamic touch, and all except one (M.H.) used their entire hands to touch the surfaces. The second question we had was whether an increase in skin contact area has an effect on discrimination performance. The results from Pont et al. (1997) provide the best comparison. The thresholds they reported for convex strips (length $20 \mathrm{~cm}$, width $2 \mathrm{~cm}$ ) placed perpendicular to the fingers or in an equivalent fashion on the knuckles or palm were between 0.76 and $1.05 / \mathrm{m}$ on average, which is almost a factor of three larger than the thresholds found in our study. In agreement with Vogels et al. (1999), we conclude that observers combine information from several parts of the hand to compare curvatures.

\section{Discrimination on Different Positions}

The third motivation for this study was to investigate the effect of spatial location on discrimination performance. There was no significant effect on thresholds of peak-topeak distance or of center position. These striking results strongly indicate that the perception of shape is independent of the position of the object. Indeed, when we handle objects in daily life, its position is hardly ever fixed: We toss the object around in our hands, pick it up from the floor, hand it to someone else, or put it on a shelf. To perform these actions correctly, it is important that we perceive the same shape, regardless of the object's position.

The position independence of thresholds enables us to infer, to some extent, the contributions made by the cutaneous and kinesthetic senses. Peak-to-peak distances covered the observer's entire arm span. The minimum peakto-peak distance was $10 \mathrm{~cm}$, which corresponded to a configuration in which both stimuli were pushed together. A distance of $70 \mathrm{~cm}$ was the maximum distance, which allowed all observers to reach both stimuli easily. For different peak-to-peak distances, positions and postures of wrists, forearms, elbows, upper arms, and shoulders varied considerably. For example, angles between hand and forearm for all observers were roughly between $205^{\circ}$ and $215^{\circ}$ (hands bent outward) at a peak-to-peak distance of $10 \mathrm{~cm}$ and between $165^{\circ}$ and $175^{\circ}$ at a distance of $70 \mathrm{~cm}$. The same holds for center positions: A shift of the stimulus set of $20 \mathrm{~cm}$ was the maximum displacement that observers could easily cover without having to twist their upper body too much. The postures of left and right arms and shoulders were different for fixed center positions, except for a center position of $0 \mathrm{~cm}$. In the case of center positions +20 or $-20 \mathrm{~cm}$, the angles between hand and 
forearm were roughly between $165^{\circ}$ and $175^{\circ}$ for one side and between $215^{\circ}$ and $225^{\circ}$ for the other.

In short, over the range of peak-to-peak distances and center positions, considerable variation occurred in the postures of left and right arms and hands, resulting in different kinesthetic stimulation. Arm movements might have also varied with stimulus position, but this is more difficult to assess. In addition, it is reasonable to assume that cutaneous stimulation was roughly the same for different positions: We did not observe the same observer using different parts of the hand to touch stimuli located at different positions. Besides, since observers could easily reach both stimuli in all positions, they were not forced to have completely different skin deformation patterns for stimuli at different positions. Note that we distinguish the cutaneous sense and kinesthesia purely in terms of function (Loomis \& Lederman, 1986): Although cutaneous mechanoreceptors in the skin might contribute to kinesthetic awareness (e.g., signaling joint position by detecting skin stretch), the function of the cutaneous sense is to provide awareness of stimulation of the outer surface of the body.

The fact that spatial positioning of the stimuli had no effect on discrimination performance then leads us to two possible conclusions: Either (1) kinesthetic stimulation does not contribute to discrimination of curved surfaces or (2) kinesthetic stimulation does contribute to discrimination, in which case a correction is made for different body postures. The studies of Pont et al. (1997, 1999) and Louw et al. (2000) provide support for the first option. Pont et al. (1999) showed that the additional arm movements made in dynamic touch, as compared with static touch, do not result in lower thresholds. Louw et al. also measured discrimination thresholds, although they did not vary stimulus position or touch mode. Their thresholds were instead measured over a broad range of stimulus dimensions in which there was considerable variation in primarily kinesthetic stimulation. They found the same dependence of thresholds on the spatial width of the Gaussian profile over the entire range of spatial scales.

\section{Discrimination Biases}

The final question we had was whether bimanual curvature discrimination biases exist. The mean of the psychometric curve is the curvature difference of the stimulus combination for which the "left"-response score was $50 \%$. An observer whose psychometric curves had nonzero means was said to show a bias. Close inspection of Figures 4, 6, and 8 indicates that several observers showed nonzero biases, whereas other observers obviously had zero biases. (Note that some observers participated in more than one experiment, and therefore appear twice or even three times in these figures.) There were also observers whose biases seemed to depend on curvature or position. In addition, biases could be either positive or negative and had different magnitudes.

If an observer's "left"-response score was $50 \%$, we assumed that the observer had not perceived any curvature difference between the two stimuli. In that case, a nonzero mean indicates that the observer showed a discrimination bias: Two physically different curvatures were perceived as being the same. As such, discrimination biases indicate that the left and the right hand transform curvature differently. However, note that a biased observer may be equally sensitive to curvature differences as an unbiased observer in terms of discrimination thresholds. It has been reported several times that biases exist in curvature perception. For example, Davidson (1972) showed that blindfolded subjects judged concave edges to be straight. In a similar fashion, observers in Henriques and Soechting's (2003) research showed small concavity biases when discriminating curved trajectories that are traced by moving the handheld vertical handle of a manipulandum in the horizontal plane. Vogels et al. (1996) reported that the "phenomenal flatness" of a flat surface was nonzero. In a study by Pont, Kappers, and Koenderink (1998), observers touching a symmetrical, doubly curved surface with one hand judged this surface to be asymmetrical. Vogels et al. (1999) found biases for the discrimination of curvature along a specified orientation of a doubly curved surface; they also found that these biases were influenced by the shape of the surface and that the effect was participant dependent.

Strictly speaking, observed biases could also be explained by a simple response bias. Irrespective of the stimulus level, the observer's response would then be biased toward one of the response alternatives, and in that case his or her response score for the subjectively equal stimulus pair would be either above or below 50\%. This bias would result in a shift of the psychometric curve, just as in the case of a discrimination bias. However, we think it unlikely that biases can be explained by response biases. The studies by Kappers et al. (1994) and Kappers and Koenderink (1996) both give strong indications for discrimination biases, for their results cannot be explained by response biases. In addition, 4 out of the 5 observers who participated in Experiment 4 had similar results in the discrimination experiments. The difference between the discrimination experiments and the matching experiment is that, in the latter, response biases are explicitly ruled out. In conclusion, although we cannot rule out response biases for individual observers, the results show that participant-dependent, bimanual discrimination biases do exist.

\section{Origin of Discrimination Biases}

Now that we know that bimanual discrimination can be biased, the question arises as to the cause of the bias. A study by Kappers, Koenderink, and Oudenaarden (1997) suggests that it probably has its origin at a central level of the nervous system. In their experiments, observers had to match the curvature of a haptically presented surface with a visually presented cross section. The systematic scaling differences between the haptic and visual perception of curvature that observers displayed were similar for both hands. This result suggests that if the left and right hands touch the same curvature under the same conditions, the signals from the two hands arriving at central levels of the nervous system are probably the same.

However, alternative explanations that focus on the peripheral nervous system are also possible. Remember that 
observers generally make upward and downward scanning movements. Suppose that the left or the right hand systematically scans the surface over a shorter distance. This difference in scanning length between left and right hand may cause an asymmetrical curvature perception. Pont et al. (1999) and Louw et al. (2000) showed that unimanual discrimination of curved strips is based primarily on the recording of slope differences. In the bimanual case, this would mean that observers record the slope difference over the surface presented to one hand and compare it with the slope difference over the surface presented to the other hand. If a stimulus is scanned over a shorter distance, the observer records a smaller slope difference, resulting in an underestimation of curvature relative to the same stimulus scanned over a larger distance. On the basis of this attitude comparison model, we have computed scanning-length differences between the two hands that would correspond to typical values of biases (see Table 1).

Scanning-length differences for biases observed in our experiments are well within the range of the stimulus dimensions. It is not easy to assess whether systematic differences in scanned areas actually existed, because the observers touched the surfaces in various ways. However, the seeming dependence of biases on spatial position in the case of individual observers may provide some evidence. If for different spatial positions observers make slightly different arm movements, the scanning length can depend on spatial position. More generally speaking, if scanningpath lengths indeed play an important role in curvature perception, there would then be an effect on everyday exploration of objects, in which observers seem to make more random movements over the surfaces. However, we must bear in mind that not only attitude differences but also curvature plays an important role in curvature discrimination, and that of course curvature is the same for different scanning lengths.

As we made clear earlier, it is desirable that more psychophysical research into the haptic perception of real-object properties be done. This article adds to the growing body of quantitative knowledge of haptic shape perception.

Table 1

Typical Values of Biases and the Corresponding ScanningLength Differences Between the Two Hands (in Centimeters)

\begin{tabular}{cccc}
\hline & \multicolumn{3}{c}{ Reference Curvature $(/ \mathrm{m})$} \\
\cline { 2 - 4 } $\operatorname{Bias}(/ \mathrm{m})$ & 1.43 & 1.83 & 2.52 \\
\hline 0.2 & 3.6 & 2.9 & 2.2 \\
0.3 & 5.1 & 4.1 & 3.2 \\
\hline
\end{tabular}

Note-Assuming that a given reference curvature was touched over the entire length of its curved surface, we first computed the slope difference over that surface. Given a certain discrimination bias, we could then compute the length of the scanning path for the subjectively equal curvature that resulted in the same slope difference. The scanning-length difference is the difference between the two scanning paths.

\section{REFERENCES}

Coren, S. (1993). The left-hander syndrome. New York: Vintage.

Davidson, P. W. (1972). Haptic judgments of curvature by blind and sighted humans. Journal of Experimental Psychology, 93, 43-55.

Garbin, C. P., \& BernsteIn, I. H. (1984). Visual and haptic perception of three-dimensional solid forms. Perception \& Psychophysics, 36, 104-110.

Goodwin, A. W., \& WheAT, H. E. (1992). Human tactile discrimination of curvature when contact area with the skin remains constant. Experimental Brain Research, 88, 447-450.

Gordon, I. E., \& MoRIson, V. (1982). The haptic perception of curvature. Perception \& Psychophysics, 31, 446-450.

Henriques, D. Y. P., \& Soechting, J. F. (2003). Bias and sensitivity in the haptic perception of geometry. Experimental Brain Research, 150, 95-108.

Kappers, A. M. L., \& KoenderinK, J. J. (1996). Haptic unilateral and bilateral discrimination of curved surfaces. Perception, 25, 739-749.

Kappers, A. M. L., Koenderink, J. J., \& Oudenaarden, G. (1997). Large scale differences between haptic and visual judgments of curvature. Perception, 26, 313-320.

Kappers, A. M. L., Koenderink, J. J., \& te Pas, S. F. (1994). Haptic discrimination of doubly curved surfaces. Perception, 23, 14831490.

Klatzky, R. L., \& Lederman, S. J. (2003). Touch. In A. F. Healy \& R. W. Proctor (Eds.), Handbook of psychology: Vol. 4. Experimental psychology (pp. 147-176). New York: Wiley.

Klatzky, R. L., Lederman, S. J., \& Metzger, V. A. (1985). Identifying objects by touch: An "expert system." Perception \& Psychophysics, 37, 299-302.

KoEnderink, J. J., \& VAn Doorn, A. J. (1992). Surface shape and curvature scales. Image \& Vision Computing, 10, 557-565.

LAKATOS, S., \& MarKs, L. E. (1999). Haptic form perception: Relative salience of local and global features. Perception \& Psychophysics, 61, 895-908.

LoOmis, J. M., \& Lederman, S. J. (1986). Tactual perception. In K. R. Boff, L. Kaufman, \& J. P. Thomas (Eds.), Handbook of perception and human performance: Vol. II. Cognitive processes and performance (pp. 31.1-31.41). New York: Wiley.

Louw, S., Kappers, A. M. L., \& Koenderink, J. J. (2000). Haptic detection thresholds of Gaussian profiles over the whole range of spatial scales. Experimental Brain Research, 132, 369-374.

Macmillan, N. A., \& Creelman, C. D. (1991). Detection theory: A user's guide. Cambridge: Cambridge University Press.

Norman, J. F., Norman, H. F., Clayton, A. M., Lianekhammy, J., \& ZIELKE, G. (2004). The visual and haptic perception of natural object shape. Perception \& Psychophysics, 66, 342-351.

Pont, S. C., Kappers, A. M. L., \& Koenderink, J. J. (1997). Haptic curvature discrimination at several regions of the hand. Perception \& Psychophysics, 59, 1225-1240.

Pont, S. C., Kappers, A. M. L., \& KoenderinK, J. J. (1998). Anisotropy in haptic curvature and shape perception. Perception, 27, 573589.

Pont, S. C., Kappers, A. M. L., \& KoenderinK, J. J. (1999). Similar mechanisms underlie curvature comparison by static and dynamic touch. Perception \& Psychophysics, 61, 874-894.

Rothwell, J. C., Traub, M. M., Day, B. L., Obeso, J. A., Thomas, P. K., \& MARSDEN, C. D. (1982). Manual motor performance in a deafferented man. Brain, 105, 515-542.

Vogels, I. M. L. C., Kappers, A. M. L., \& Koenderink, J. J. (1996). Haptic aftereffect of curved surfaces. Perception, 25, 109-119.

Vogels, I. M. L. C., Kappers, A. M. L., \& Koenderink, J. J. (1999). Influence of shape on haptic curvature perception. Acta Psychologica, 100, 267-289.

Wichmann, F. A., \& Hill, N. J. (2001). The psychometric function: II. Bootstrap-based confidence intervals and sampling. Perception \& Psychophysics, 63, 1314-1329. 


\section{APPENDIX}

We fit the psychometric curves to the data by means of a maximum-likelihood fitting procedure. The likelihood is the probability that a set of observations will occur, given a particular set of parameters. By maximizing the likelihood, we find the best-fit parameters. Contrary to the least-squares method, this procedure takes into account the binomial distribution of the data. The likelihood is the product of the chances of each individual data point:

$$
L=\prod_{i=1}^{m} P\left(\text { datapoint }_{i} / \text { parameters }\right) .
$$

In the case of a two-alternative forced choice experiment, the chance $P$ is binomially distributed with the psychometric function $\phi$ giving for each stimulus pair $x_{i}$ the probability of a correct response: $p_{i}=\phi\left(x_{i} \mid \mu, \sigma\right)$. If $n_{i}$ is the number of times the $i$ th point on the psychometric curve has been sampled and $y_{i}$ is the number of "left" responses for curvature difference $x_{i}$, the likelihood then becomes

$$
L(\mu, \sigma)=\prod_{i=1}^{m}\left(\begin{array}{l}
n_{i} \\
y_{i}
\end{array}\right) p_{i}^{y_{i}}\left(1-p_{i}\right)^{n_{i}-y_{i}} .
$$

Maximization of this function yields best-fit estimates of parameters $\mu$ and $\sigma$. Finally, we estimated standard errors using a parametric bootstrap procedure (Wichmann \& Hill, 2001). We simulated 100 random data sets using the original best-fit parameters $\mu$ and $\sigma$, fitted psychometric curves again, and calculated the standard deviations of the collections of $\mu \mathrm{s}$ and $\sigma \mathrm{s}$. These standard deviations were taken as the standard errors for both parameters.

(Manuscript received April 1, 2005;

revision accepted for publication October 19, 2005.) 